\title{
Acute disseminated encephalomyelitis following endonasal resection of a craniopharyngioma: illustrative case
}

\author{
Konrad Gag, ${ }^{1}$ Jonas Müller, MD, ${ }^{2}$ Marie Süße, MD, ${ }^{1}$ Robert Fleischmann, MD, ${ }^{1}$ and Henry W. S. Schroeder, MD
}

Departments of ${ }^{1}$ Neurology and ${ }^{2}$ Neurosurgery, University Medicine Greifswald, Greifswald, Germany

BACKGROUND Acute disseminated encephalomyelitis (ADEM) is a rare, acquired demyelination syndrome that causes cognitive impairment and focal neurological deficits and may be fatal. The potentially reversible disease mainly affects children, often after vaccination or viral infection, but may be seen rarely in adults.

OBSERVATIONS A 50-year-old woman presented with loss of visual acuity of the left eye. Magnetic resonance imaging (MRI) revealed an intra- and suprasellar mass, which was removed successfully. On postoperative day 1, MRI showed gross total resection of the lesion and no surgery-related complications. On postoperative day 2, the patient presented with a progressive left-sided hemiparesis, hemineglect, and decline of cognitive performance. MRI showed white matter edema in both hemispheres. Cerebrospinal fluid analysis revealed mixed pleocytosis $(355 / \mu \mathrm{L})$ without further evidence of infection. In synopsis of the findings, ADEM was diagnosed and treated with intravenous immunoglobulins. Shortly thereafter, the patient recovered, and no sensorimotor deficits were detected in the follow-up examination.

LESSONS Pituitary gland pathologies are commonly treated by transsphenoidal surgery, with only minor risks for complications. A case of ADEM after craniopharyngioma resection has not been published before and should be considered in case of progressive neurological deterioration with multiple white matter lesions.

https://thejns.org/doi/abs/10.3171/CASE21293

KEYWORDS acute disseminated encephalomyelitis; ADEM; acquired demyelination syndrome; cerebrospinal fluid; pituitary surgery

Acute disseminated encephalomyelitis (ADEM) is a rare, acquired demyelination syndrome that causes cognitive impairment and focal neurological deficits and may be fatal. This case describes the first mention of ADEM as a complication of a craniopharyngioma resection.

\section{Illustrative Case}

A 50-year-old woman presented to our neurosurgical outpatient clinic with loss of visual acuity and an enlarged blind spot of the left eye. On magnetic resonance imaging (MRI), a partially hemorrhaged pituitary adenoma was suspected $(13 \times 13 \mathrm{~mm})$ (Fig. $1 \mathrm{~A}-\mathrm{C})$. A treatment attempt with cabergoline ( $0.5 \mathrm{mg}$ every third day in the 12 days before surgery) had already been made under the suspicion of a prolactinoma because of an elevated prolactin level $(1,541 \mathrm{mlU} / \mathrm{L}$ [reference range
102-496 mlU/L]). Because an insufficient effect was seen under this treatment, surgical removal was indicated. The lesion was removed via an endoscopic endonasal binostril approach. During tumor resection, the paper-thin diaphragm ruptured and a major cerebrospinal fluid (CSF) leak occurred. Intraoperatively, it became clear that the lesion was a cystic craniopharyngioma. After tumor removal, the skull base defect was closed with fat taken from the belly. Surgery and anesthesia went well, with no complications. The patient woke up without any new neurological deficits and was transferred to our intensive care unit (ICU) for overnight observation. MRI performed on postoperative day 1 confirmed a gross total tumor resection without complications (Fig. 1D-F).

On postoperative day 2, however, the patient developed a rapidly progressive left hemiparesis (upper extremity: Medical Research

ABBREVIATIONS ADEM = acute disseminated encephalomyelitis; $C N S$ = central nervous system; $C S F$ = cerebrospinal fluid; ICU = intensive care unit; IVIG = intravenous immunoglobulin; MRC = Medical Research Council; MRI = magnetic resonance imaging; OCB = oligoclonal bands; ODS = osmotic demyelination syndrome; PRES = posterior reversible encephalopathy syndrome; SARS-CoV-2 = severe acute respiratory syndrome coronavirus. INCLUDE WHEN CITING Published October 18, 2021; DOI: 10.3171/CASE21293.

SUBMITTED May 10, 2021. ACCEPTED May 27, 2021.

(C) 2021 The authors, CC BY-NC-ND 4.0 (http://creativecommons.org/licenses/by-nc-nd/4.0/). 

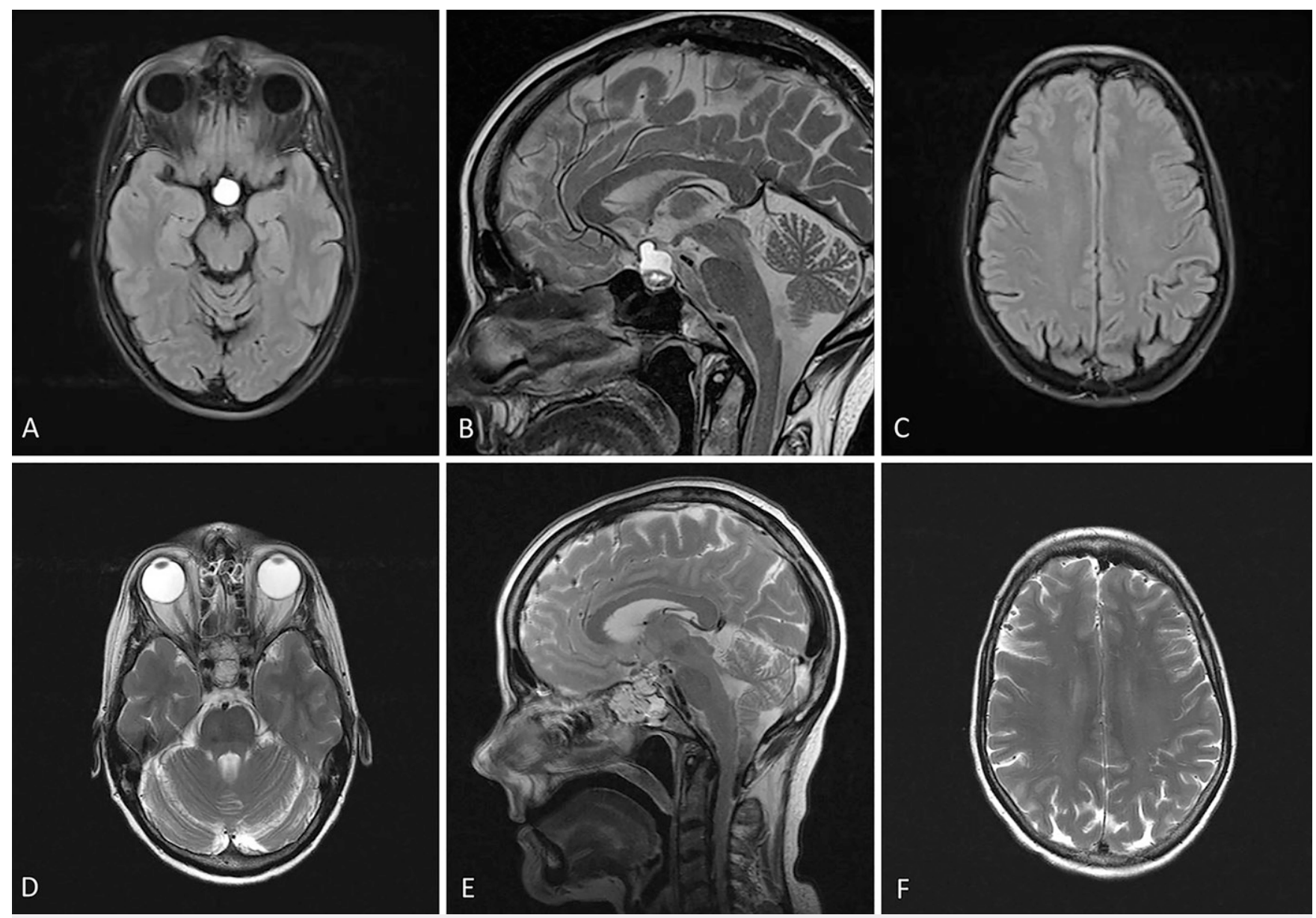

FIG. 1. A-C: MRI obtained before surgery. A and C: Axial fluid-attenuated inversion recovery (FLAIR) images. B: Sagittal T2-weighted image showing a $13 \times 13$-mm pituitary mass. D-F: MRI obtained 1 day after surgery. T2-weighted images showing gross tumor resection with fat seal in place.

Council [MRC] scale 0/5; lower extremity: MRC scale 2/5) with hemineglect and altered state of consciousness. MRI showed extensive white matter edema. The white matter lesions were most prominent in the parietal and frontal lobe of the right hemisphere but involved both sides. There was no cortical involvement. Atypical posterior reversible encephalopathy syndrome (PRES) was radiologically assumed because of the pattern of the white matter edema (Fig. 2A-C). However, an infectious origin, such as progressive multifocal leukoencephalopathy, ADEM, or osmotic demyelination syndrome (ODS), was also included in the differential diagnosis. The initial treatment consisted of intravenous fluid infusion and lowering of high blood pressure when necessary. During the patient's stay in the ICU, we recorded slightly elevated blood pressures (Fig. 3). We did not observe seizures, nor did the daily clinical examination or ophthalmological examination with perimetric testing on days 1 and 15 indicate any worsening of vision. Because of lack of hypertension and suspected correlation with the procedure, atypical PRES as the causative factor was soon rejected.

In the meantime, pathology confirmed a craniopharyngioma World Health Organization ${ }^{\circ} \mathrm{l}$. A spinal tap on postoperative day 8 was performed to exclude an infectious or autoimmune origin of the white matter edema. It showed pleocytosis (335 leukocytes/ $\mu \mathrm{L}$ [reference range: $<5 / \mu \mathrm{L}$; $44 \%$ neutrophils, $47 \%$ lymphocytes), an elevated lactate concentration (lactate: $4.4 \mathrm{mmol} / \mathrm{L}$ [reference range: $<2.5 \mathrm{mmol} / \mathrm{L}$ ]), an elevated concentration of total protein $(614 \mathrm{mg} / \mathrm{L}$ [reference range: $<500 \mathrm{mg} / \mathrm{L}]$ ), and oligoclonal bands (OCBs) isolated in the CSF (Table 1). The pathological, microbiological, and virological diagnostics regarding human immunodeficiency virus, herpes simplex virus, varicella zoster virus, Epstein Barr virus, human polyoma virus 1, John Cunningham virus, and bacterial infection were negative. Because of suspected perioperative infection based on CSF analysis, the patient empirically received antibiotic treatment with vancomycin (1,000 $\mathrm{mg}$ every 12 hours) and meropenem ( $250 \mathrm{mg} / \mathrm{h}$ continuously) for 48 hours. Another spinal tap or cerebral biopsy was not performed because the patient did not consent.

The patient did not show clinical or radiological improvement under antibiotic treatment, which favored the differential diagnosis of ADEM. Therefore, we continued ongoing treatment with hydrocortisone, which was routinely initiated during surgery as a standard protocol for pituitary surgery. Further desmopressin applications, which were initially necessary because of high diuresis and rising sodium levels, were avoided.

Beginning on day 10 , we decided to treat primarily with overall $2 \mathrm{~g} / \mathrm{kg}$ intravenous immunoglobulin (IVIG) for 3 days. High-dose corticosteroids were not used so as not to interfere with wound healing and avoid further homeostatic disturbances. After 3 days of treatment, the patient showed improvement of hemiparesis and neglect in clinical examination (upper extremity: MRC scale 0/5; lower extremity: MRC 

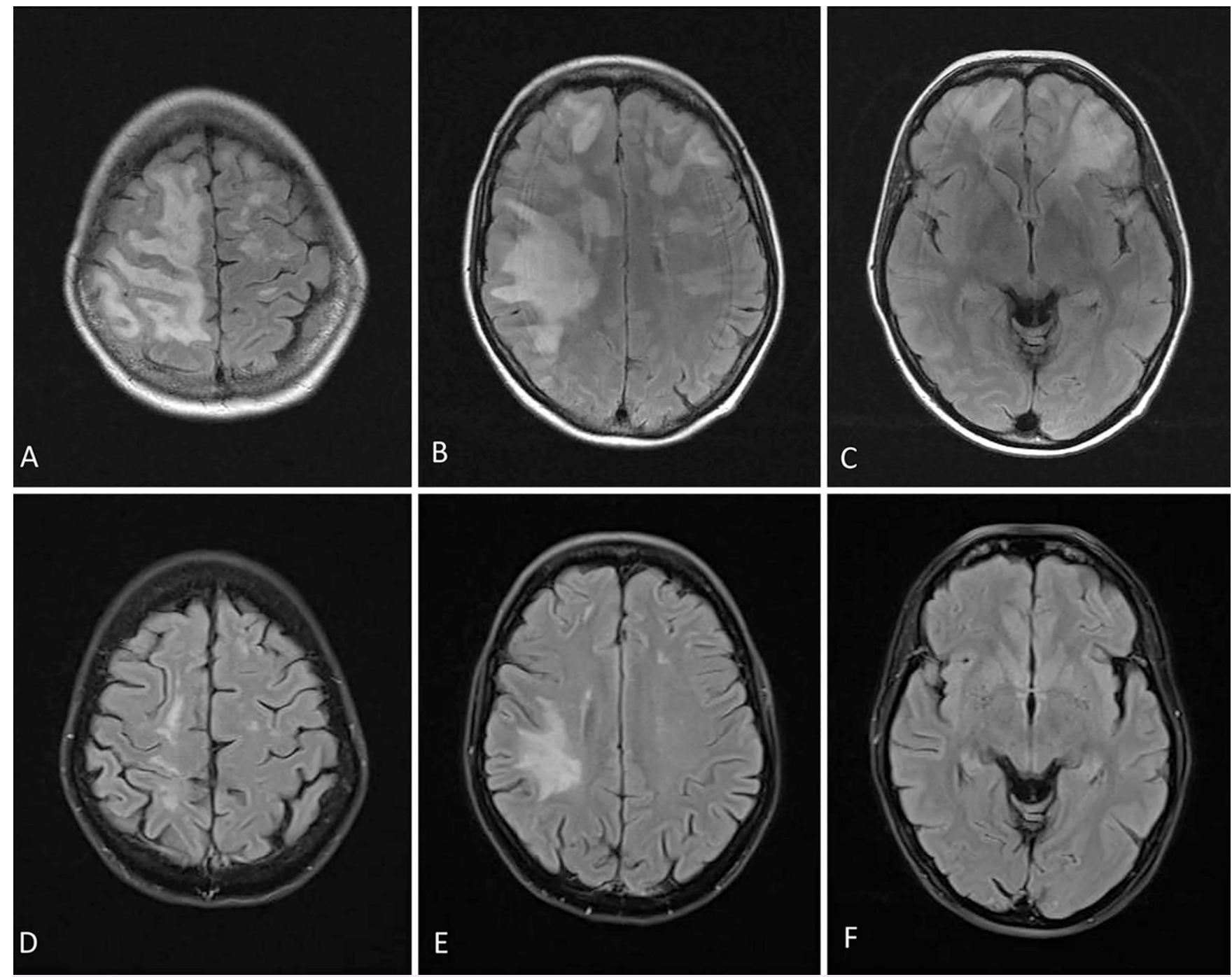

FIG. 2. A-C: MRI obtained 3 days after surgery. Axial FLAIR images showing multiple subcortical hyperintense lesions in both cerebral hemispheres. D-F: MRI obtained 94 days after surgery. Axial FLAIR images showing a marked but incomplete resolution of the lesions.

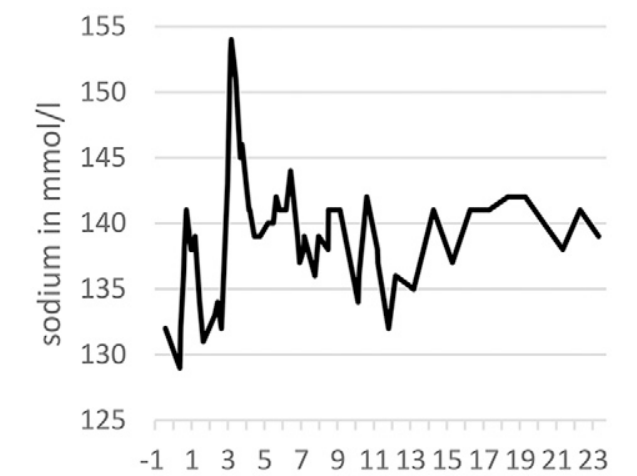

A

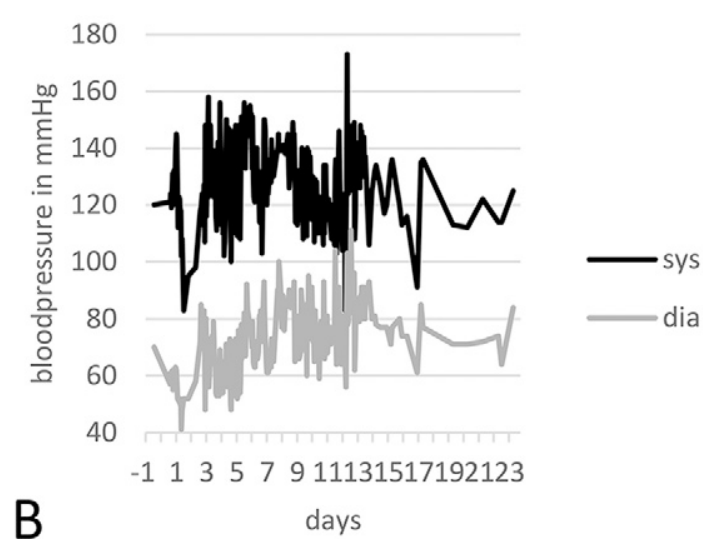

FIG. 3. A: Initial mild hyponatremia with postoperative increase on day 3. B: Recorded blood pressure values during residency. 
TABLE 1. CSF lab values on day 8 after surgery

\begin{tabular}{lc}
\hline \multicolumn{1}{c}{ CSF Lab } & Value \\
\hline Lactate (reference range: $0.6-2.5 \mathrm{mmol} / \mathrm{L}$ ) & $4.4 \mathrm{mmol} / \mathrm{L}$ \\
\hline Total protein (reference range: $<500 \mathrm{mg} / \mathrm{L}$ ) & $614 \mathrm{mg} / \mathrm{L}$ \\
\hline Erythrocytes (reference range: $0 / \mu \mathrm{L}$ ) & $59 / \mu \mathrm{L}$ \\
\hline Leukocytes (reference range: $<5 / \mu \mathrm{L}$ ) & $355 / \mu \mathrm{L}$ \\
\hline Lymphocytes & $47 \%$ \\
\hline Neutrophil granulocytes & $44 \%$ \\
\hline Activated lymphocytes & $3 \%$ \\
\hline OCB & Isolated in CSF \\
\hline
\end{tabular}

scale 4/5). She could be transferred to the neurosurgical ward on postoperative day 13 . MRI revealed declining subcortical edema, and the patient was transferred to the rehabilitation clinic on postoperative day 23.

She presented to the outpatient clinic with results from MRI (Fig. 2D-F) performed on day 94, and the treating clinician noted a subjective reduced cognitive performance without any further neurological symptoms (Barthel scale 100/100; Modified Rankin Scale 1/6).

\section{Discussion}

\section{Observations}

We present the first case of ADEM after craniopharyngiomal resection. $A D E M$ is a single, multifocal inflammatory demyelination disorder of the central nervous system (CNS) and is described as one of the acquired demyelination syndromes. ADEM is a rare disease (incidence of $0.07-0.9 / 100,000$ ) and is seen mainly in children (median age 5-8 years). It is associated with prodromal viral infections, vaccinations, and, although uncommon, bacterial infections in up to $67 \%$. ${ }^{1-4}$ Mild pleocytosis in CSF (usually $<100 / \mu \mathrm{L}$, although according to reports up to $270 / \mu \mathrm{L}$ ) is frequent in up to $73 \%$ of adults, whereas OCBs are rarely detected (in only $28 \%$ of adult patients with ADEM) and may disappear over time. ${ }^{5-7}$

The first neurological deficits occur usually after 2 to 21 days. ${ }^{1}$ The pathogenetic mechanism is understood to involve an autoimmune reaction against myelin proteins (e.g., myelin basic protein) as a form of a molecular mimicry. ${ }^{8-10}$ Another theory based on animal experiments describes ADEM as an inflammatory response with vascular congestion and increased permeability of CNS vasculature facilitating antigen

\section{TABLE 2. Revised ADEM criteria for children}

\begin{tabular}{l}
\hline $\begin{array}{l}\text { First polyfocal, clinical CNS event with presumed inflammatory } \\
\text { demyelinating cause }\end{array}$ \\
\hline Encephalopathy (not explained by fever) \\
\hline No new findings (clinical \& MRI) $\geq 3$ mos after onset \\
\hline Abnormal MRI during the acute phase ( 3 mos) \\
\hline MRI conspicuities \\
\hline Typical: diffuse, large $(>1-2 \mathrm{~cm}$ ) lesions involving predominantly \\
cerebral white matter \\
Seldom: T1 hypointense lesions in white matter \\
Possible: deep gray matter lesions (e.g., thalamus or basal \\
ganglia)
\end{tabular}

Data obtained from Krupp et al., 2013. exposure. $^{10}$ This is thought to induce an inflammatory cascade of edema, infiltration of inflammatory cells, and perivenous hemorrhage, which results in demyelination, gliosis, and necrosis. ${ }^{10}$ Although more frequently detected in children, ADEM can also rarely occur in adults. As a result of the rarity of the disease in adults, no international definition for ADEM in adults exists. Clinical and radiological criteria were revised for children by Krupp et al. and include the criteria shown in Table $2{ }^{4}$

Treatment suggestions derive from expert opinions and observational studies and propose a high dosage of intravenous methylprednisolone for 3 to 5 days followed by oral administration of corticosteroids with gradual dosage reduction for 4 to 6 weeks. IVIG or plasma exchange is considered second-line therapy for steroid-unresponsive ADEM. ${ }^{1,11}$ Even with early initiation of therapy, up to $35 \%$ of patients have permanent deficits. The mortality rate in children is $1 \%-3 \%$, but in adults it is much higher at $25 \%{ }^{11,12}$ Long-term multicenter studies for evaluation of treatment recommendations and clinical outcome are thus needed. Because it is not always possible to force pathological confirmation with a brain biopsy, diseases that come into question for differential diagnosis must often be excluded in the diagnostic process, particularly infections (especially when CSF pleocytosis $>100 / \mu \mathrm{L}$ is present). Therefore, initial empirical treatment with antibiotics and extensive diagnostics is recommended. ${ }^{1,5}$ First occurrence of other autoimmune diseases, such as multiple sclerosis, can be hard to differentiate and is sometimes only distinguishable during the course of the disease. ${ }^{5}$

Other differential diagnoses in our case involved atypical PRES and ODS. PRES typically presents with headache, seizures, altered mental state, and visual loss. It is often accompanied by severe hypertension (>150-160 mm Hg), which exceeds the autoregulatory limits of the brain and leads to capillary bed injury, fluid egress, and vasogenic edema. ${ }^{13}$ PRES manifestations typically involve the parieto-occipital subcortical substance $(53 \%)$ with the above-mentioned symptoms. In rarer cases, PRES may manifest in other locations (superior frontal sulcus [17\%], central [14\%], and holohemispheric watershed [17\%]). ${ }^{14}$ In our patient, the diagnosis of atypical PRES was rejected because of permanent normal systolic blood pressure (Fig. 3), higher cell count in CSF, which is not described in PRES (pleocytosis in PRES up to $41 / \mu \mathrm{L}$ ), and the atypical MRI pattern (Fig. $2 \mathrm{~A}-\mathrm{C}){ }^{14-16}$ Extrapontine myelinolysis as part of ODS is mostly localized in the basal ganglia and cerebellum and is associated with extrapyramidal motor symptoms $(60 \%)$. The risk of fast compensating hyponatremia $\left(>12 \mathrm{mmol} / 24 \mathrm{~h}\right.$ ) may end in a fatal ODS. ${ }^{17}$ Although the sodium level rose approximately $20 \mathrm{mmol} / 20 \mathrm{~h}$ between days 3 and 4 in our patient, the brain edema and clinical symptoms revolved before this osmotic disturbance. All in all, the atypical localization of the brain edema not involving typical structures of ODS and the missing temporal association with compensation of hyponatremia make ODS implausible. ${ }^{17}$

This report describes the first case of ADEM as a complication of craniopharyngiomal resection. Although no cerebral biopsy was performed, the neurological symptoms and MRI characteristics fit the consensus diagnostic criteria. ${ }^{4}$ Relevant differential diagnoses were excluded in the diagnostic workup. The excellent outcome of the patient with one cycle of IVIG administration further supports the diagnosis of ADEM. ${ }^{1}$ We hypothesize that the patient is equipped with a certain immunological susceptibility and reacted to an antigen presentation either displaced based on the transnasal approach or through intraoperative microtrauma during tumor resection. We have no knowledge of infections or vaccinations before the operation as a potential trigger mechanism. ${ }^{3}$ Current case reports describe ADEM 
as a consequence of severe acute respiratory syndrome coronavirus (SARS-CoV-2) infection. ${ }^{18}$ However, our patient tested negative for SARS-CoV-2 twice, so there is no evidence of a link to a previous infection. In our opinion, minor discrepancies do not contradict our reasonable suspicion. The detected OCBs are found in $10 \%-29 \%$ of $\mathrm{ADEM}$, and the unusually high pleocytosis may have originated with

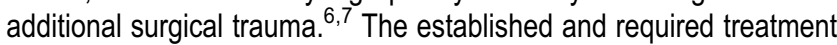
with desmopressin after pituitary gland surgery may have additionally increased ADEM, as suggested by animal studies. ${ }^{19}$

There is only one comparable case report of ADEM, which occurred after a third ventricle colloid cyst resection. The authors suspected that the ultrasonic device, in our case not used, spread cyst content, which resulted in a possible inflammatory response. ${ }^{20}$ Nevertheless, there may be an unknown but similar pathogenic pathway. Surprisingly, there are even cases of ADEM noted after appendectomy, prostate resection, and cranial aneurysm coiling, suggesting unknown independent risk factors in surgical procedures. ${ }^{21-23}$

\section{Lessons}

This case report shows the first described case of ADEM as a complication of a pituitary gland procedure with fast improvement under IVIG therapy. Even under the recommended treatment, ADEM still results potentially in lifelong physical and cognitive impairment. The occurrence of ADEM as a rare periprocedural neurosurgical complication with possible fatal consequences shows the importance of not only alerting neurosurgeons and critical care physicians but also extensively investigating the linkage and pathophysiology of ADEM in adults in the future. The research objective should also be to improve treatment and avoid these uncommon complications.

\section{References}

1. Paolilo RB, Deiva K, Neuteboom R, Rostásy K, Lim M. Acute disseminated encephalomyelitis: current perspectives. Children (Basel). 2020;7(11):210.

2. Berzero G, Cortese A, Ravaglia S, Marchioni E. Diagnosis and therapy of acute disseminated encephalomyelitis and its variants. Expert Rev Neurother. 2016;16(1):83-101.

3. Koelman DL, Mateen FJ. Acute disseminated encephalomyelitis: current controversies in diagnosis and outcome. J Neurol. 2015;262(9):2013-2024.

4. Krupp LB, Tardieu M, Amato MP, et al. International Pediatric Multiple Sclerosis Study Group criteria for pediatric multiple sclerosis and immune-mediated central nervous system demyelinating disorders: revisions to the 2007 definitions. Mult Scler. 2013;19(10): 1261-1267.

5. Koelman DL, Chahin S, Mar SS, et al. Acute disseminated encephalomyelitis in 228 patients: a retrospective, multicenter US study. Neurology. 2016;86(22):2085-2093.

6. Dale RC, de Sousa C, Chong WK, Cox TC, Harding B, Neville BG. Acute disseminated encephalomyelitis, multiphasic disseminated encephalomyelitis and multiple sclerosis in children. Brain. 2000;123(Pt 12):2407-2422.

7. Franciotta $D$, Columba-Cabezas $S$, Andreoni L, et al. Oligoclonal IgG band patterns in inflammatory demyelinating human and mouse diseases. J Neuroimmunol. 2008;200(1-2):125-128.

8. Murthy JM. Acute disseminated encephalomyelitis. Neurol India. 2002;50(3):238-243.

9. Wucherpfennig KW, Strominger JL. Molecular mimicry in T cellmediated autoimmunity: viral peptides activate human $T$ cell clones specific for myelin basic protein. Cell. 1995;80(5): 695-705.
10. Katz-Levy Y, Neville KL, Girvin AM, et al. Endogenous presentation of self myelin epitopes by CNS-resident APCs in Theiler's virusinfected mice. J Clin Invest. 1999;104(5):599-610.

11. Pohl D, Alper G, Van Haren K, et al. Acute disseminated encephalomyelitis: updates on an inflammatory CNS syndrome. Neurology. 2016;87(9 suppl 2):S38-S45.

12. Sonneville R, Demeret S, Klein I, et al. Acute disseminated encephalomyelitis in the intensive care unit: clinical features and outcome of 20 adults. Intensive Care Med. 2008;34(3):528-532.

13. Saad AF, Chaudhari R, Wintermark M. Imaging of atypical and complicated posterior reversible encephalopathy syndrome. Front Neurol. 2019;10:964.

14. Liman TG, Bohner G, Heuschmann PU, Endres M, Siebert E. The clinical and radiological spectrum of posterior reversible encephalopathy syndrome: the retrospective Berlin PRES study. J Neurol. 2012;259(1):155-164.

15. Ellis CA, McClelland AC, Mohan S, et al. Cerebrospinal fluid in posterior reversible encephalopathy syndrome: implications of elevated protein and pleocytosis. Neurohospitalist. 2019;9(2):58-64.

16. Neeb L, Hoekstra J, Endres M, Siegerink B, Siebert E, Liman TG. Spectrum of cerebral spinal fluid findings in patients with posterior reversible encephalopathy syndrome. J Neurol. 2016;263(1):30-34.

17. Lambeck J, Hieber M, Dreßing A, Niesen WD. Central pontine myelinosis and osmotic demyelination syndrome. Dtsch Arztebl Int. 2019;116(35-36):600-606.

18. Novi G, Rossi T, Pedemonte E, et al. Acute disseminated encephalomyelitis after SARS-CoV-2 infection. Neurol Neuroimmunol Neuroinflamm. 2020;7(5):e797.

19. Quintanar-Stephano A, Organista-Esparza A, Chavira-Ramírez R, Kovacs K, Berczi I. Effects of neurointermediate pituitary lobectomy and desmopressin on acute experimental autoimmune encephalomyelitis in Lewis rats. Neuroimmunomodulation. 2012;19(3):148-157.

20. Desse N, Sellier A, Bernard C, Dagain A. Fatal acute disseminated encephalomyelitis (ADEM) after third ventricle colloid cyst resection with ultrasonic aspirator during neuroendoscopic procedure. Acta Neurochir (Wien). 2018;160(9):1789-1792.

21. White LD, White LK, Baxter DF, Melhuish TM. Adult onset acute disseminated encephalomyelitis following appendicitis: a case report. J Neurol Neurosci. 2016;7:S3.

22. Ceronie B, Cockerell OC. Acute disseminated encephalomyelitis in an older adult following prostate resection. eNeurologicalSci. 2018;14:40-42.

23. Deus-Silva L, Lum C, De Meulemeester C, dos Santos MP. Severe aggressive acute disseminated encephalomyelitis-like reaction after aneurysm coiling. Neurosurgery. 2010;66(1):E222-E223.

\section{Disclosures}

The authors acknowledge support for the article processing charge from the DFG (German Research Foundation, 393148499) and the Open Access Publication Fund of the University of Greifswald.

\section{Author Contributions}

Conception and design: Gag, Fleischmann. Acquisition of data: Gag, Fleischmann. Analysis and interpretation of data: Gag, Süße, Fleischmann. Drafting the article: Gag, Süße. Critically revising the article: Müller, Süße, Schroeder. Reviewed submitted version of manuscript: Süße, Fleischmann, Schroeder. Administrative/technical/ material support: Gag, Schroeder.

\section{Correspondence}

Konrad Gag: University Medicine Greifswald, Greifswald, Germany. konrad.gag@med.uni-greifswald.de. 